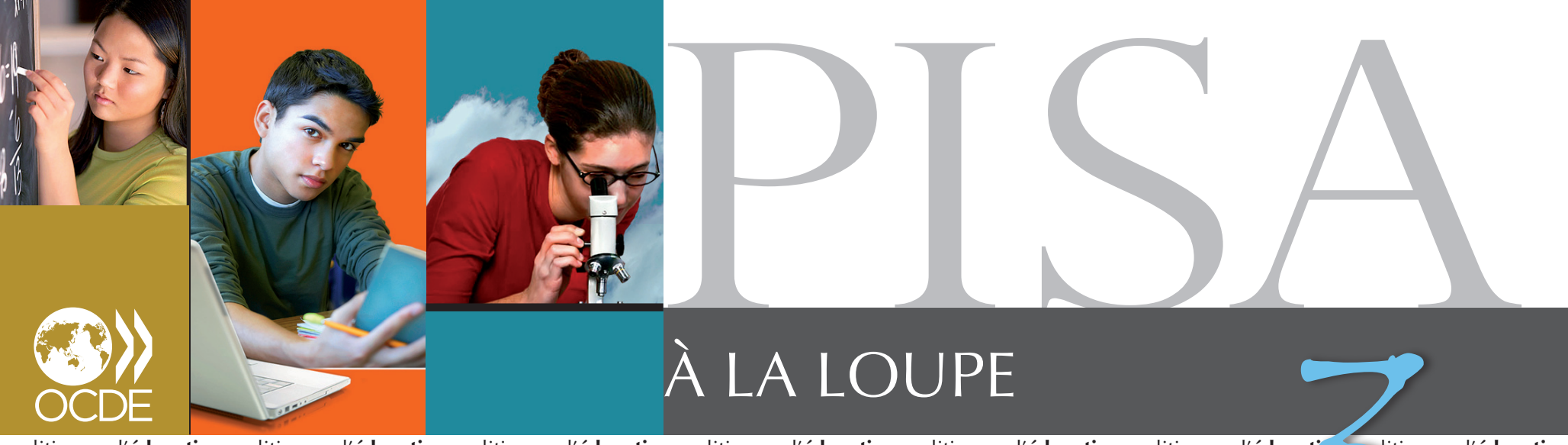

politiques d'éducation politiques d'éducation politiques d'éducation politiques d'éducation politiques d'éducation politiques d'éducation politiques d'éducation

\title{
Faut-il investir dans les cours de soutien après la classe?
}

- Dans les pays qui obtiennent de bons résultats au PISA, les élèves passent moins de temps, en moyenne, dans les cours supplémentaires après la classe et l'étude individuelle, et plus de temps en classe que les élèves des pays qui obtiennent de moins bons résultats.

- Les cours supplémentaires dispensés par un enseignant de l'établissement sont un facteur d'équité, tandis que les cours donnés par un enseignant qui n'est pas de l'établissement peuvent au contraire accroître l'inégalité entre élèves.

- Lorsque les élèves sont convaincus de l'importance d'avoir de bons résultats en sciences, le temps passé en classe exerce un impact plus grand sur leurs performances dans cette matière.

C'est bien la qualité Dans un contexte de concurrence féroce pour rentrer dans les du temps d'apprentissage, bonnes universités et décrocher les bons emplois, les élèves de et non la quantité, l'enseignement secondaire sont souvent encouragés à suivre des cours qui fait la différence. supplémentaires après la classe dans les matières déjà enseignées si cela suppose de renoncer à d'autres façons amusantes et intéressantes d'occuper leur temps libre après la classe, comme de faire du sport, de prendre des cours de musique ou de travailler bénévolement dans un centre ou hôpital de la communauté. Dans les pays de l'OCDE, les élèves passent, en moyenne, près de deux heures et demie par semaine dans des cours supplémentaires après la classe. En Corée, en Grèce, en Israël et en Turquie, et dans les pays partenaires, en Azerbaïdjan, au Kirghizistan, au Qatar et en Tunisie, ils y passent même plus de quatre heures et demie. Cet investissement dans les cours de soutien est-il rentable?

Pas toujours. À vrai dire, si l'on en croit les résultats de l'enquête PISA 2006, le temps d'apprentissage passé dans les cours de perfectionnement après la classe et l'étude individuelle présente une corrélation négative avec la performance. Bien entendu, l'on pourrait arguer que les élèves qui suivent ces cours le font pour améliorer leurs résultats, plutôt que pour consacrer davantage de temps à leurs études. Pourtant, dans tous les pays, les résultats de l'enquête montrent que les élèves tendent à obtenir de meilleurs résultats si une forte proportion de leur temps d'apprentissage total - qui comprend les cours normaux, les cours supplémentaires après la classe et l'étude individuelle - est passée en cours normaux dans le cadre scolaire. Par exemple, en Australie, en Finlande, au Japon et en Nouvelle-Zélande, pays tous très performants, plus de $70 \%$ de l'apprentissage des sciences se déroule durant la classe. Toutefois, le temps passé à apprendre ne peut expliquer à lui seul pourquoi les élèves de ces pays sont parmi les plus performants. En effet, dans tous ces pays excepté la Nouvelle-Zélande, les élèves de 15 ans passent moins de temps à apprendre les sciences que la moyenne des pays de l'OCDE. Le même schéma s'observe pour le temps d'apprentissage dédié à la culture mathématique et à la langue d'instruction, ce qui suppose que c'est bien la qualité des cours, et non la quantité d'heures d'apprentissage, qui exerce l'impact le plus signifiant sur la performance des élèves. 


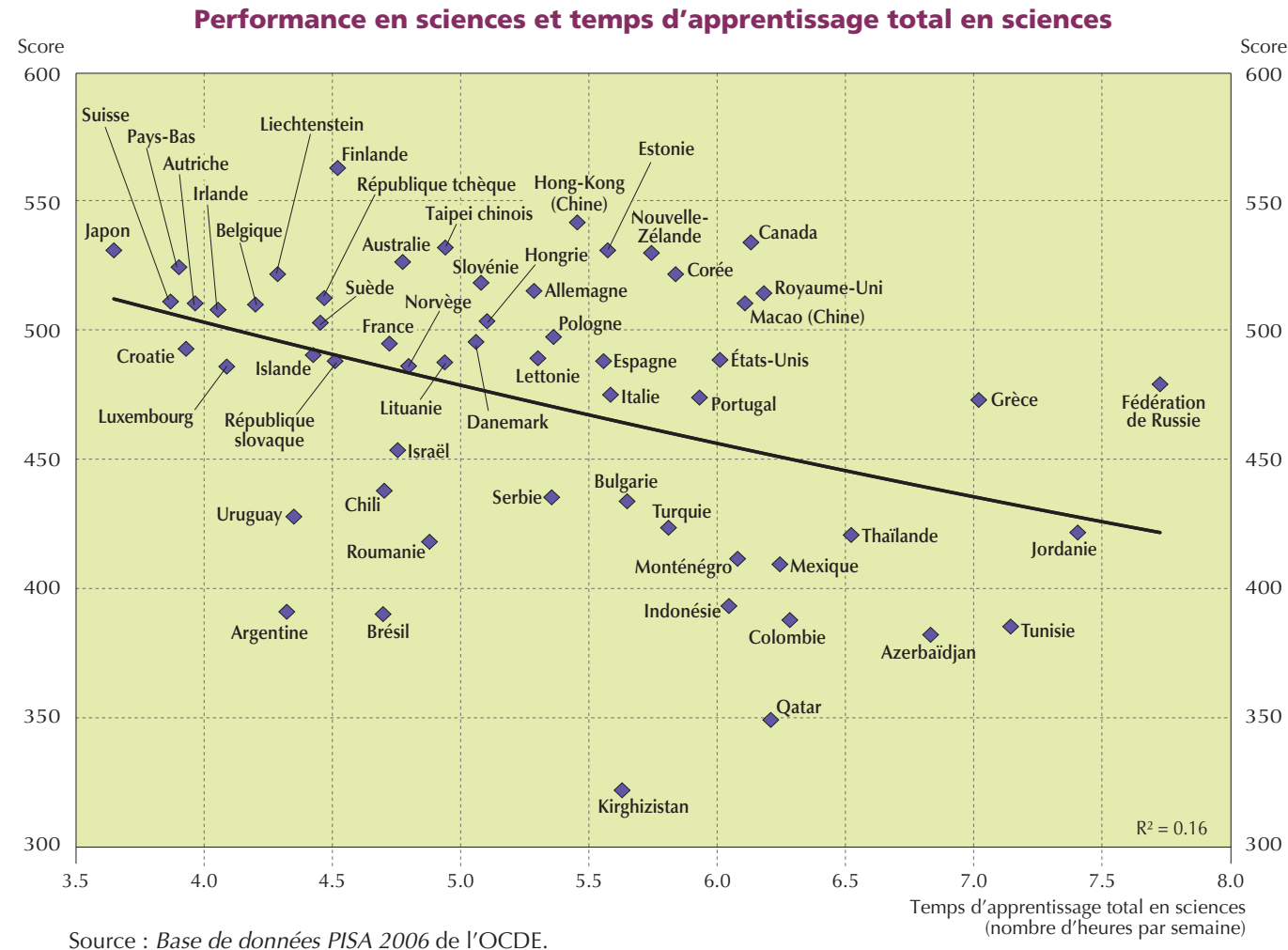

Source : Base de données PISA 2006 de l'OCDE.

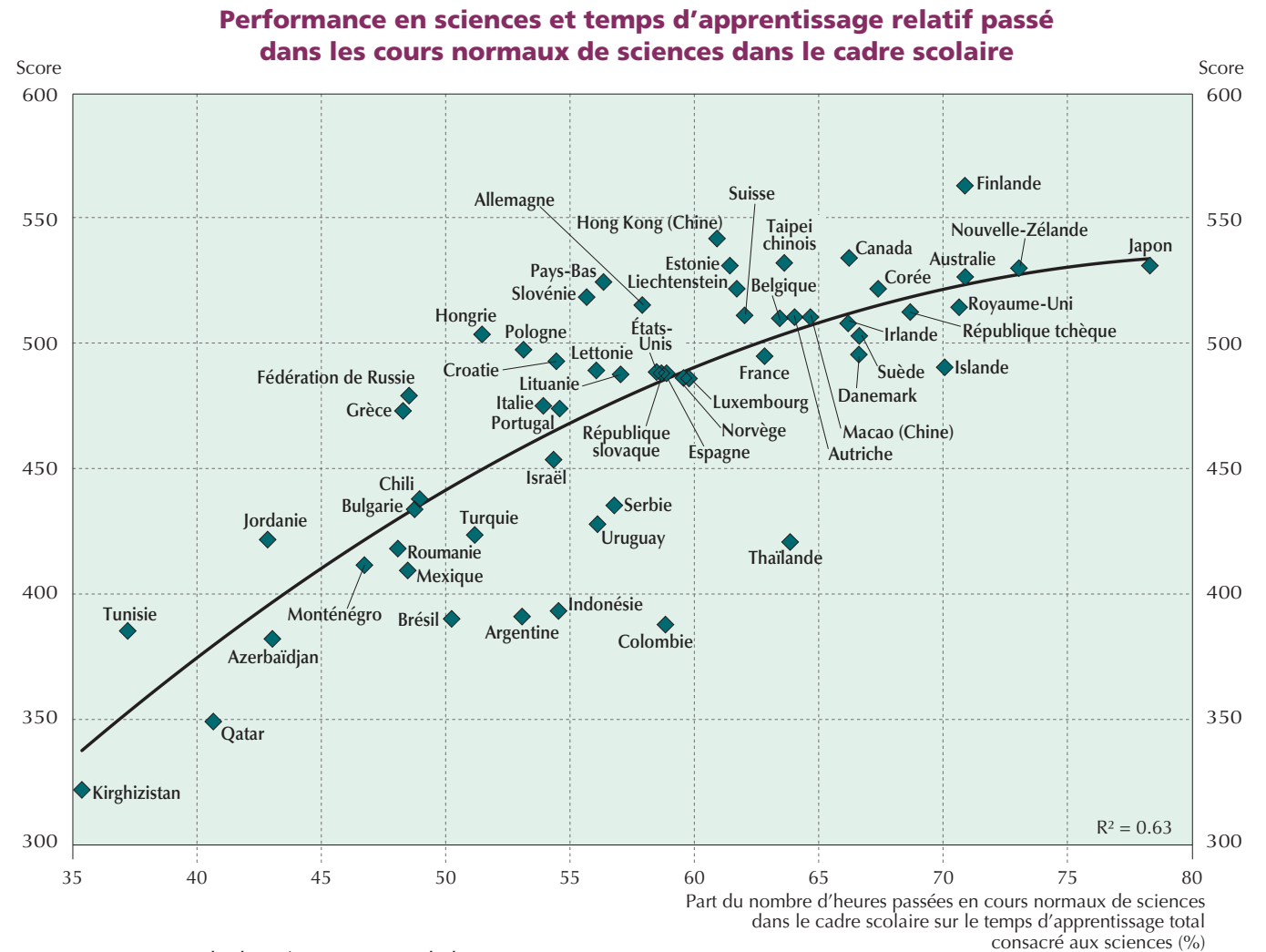

Source : Base de données PISA 2006 de l'OCDE. 
Certains cours supplémentaires après la classe sont plus inclusifs que d'autres.

Les types et finalités des cours supplémentaires après la classe varient fortement, depuis les cours dispensés dans l'établissement scolaire de l'élève par un enseignant de cet établissement jusqu'à ceux dispensés en dehors des locaux de l'établissement par un éducateur non affilié à cet établissement, en passant par les cours de " rattrapage » et les cours de perfectionnement. Si les résultats du PISA montrent que certains types de cours supplémentaires après la classe permettent d'obtenir de meilleurs résultats, la mesure dans laquelle la participation à ces cours modère ou renforce les inégalités existantes entre les élèves de différents groupes socio-économiques varie d'un pays à l'autre. Par exemple, en Corée, en Italie, en Pologne et au Royaume-Uni, et dans les pays et économies partenaires, en Lituanie, en Fédération de Russie et au Taipei chinois, la participation à des cours supplémentaires après la classe dispensés par un enseignant de l'établissement a tendance à réduire l'impact du milieu socio-économique des élèves sur leur performance scolaire. En effet, les élèves défavorisés sont davantage susceptibles de participer à ce type de cours après la classe et donc de progresser par rapport aux élèves qui n'y participent pas. En revanche, en Corée, en Estonie, en Espagne, en Irlande, en Pologne et en Turquie, et dans les pays et économies partenaires, en Bulgarie, à Hong-Kong (Chine), au Kirghizistan, en Lettonie, au Taipei chinois et en Uruguay, les leçons en groupe dispensées par un enseignant qui n'appartient pas à l'établissement ont tendance à renforcer l'impact du milieu socio-économique sur la performance. Dans ces pays, il apparaît que les élèves issus d'un milieu socio-économique favorisé ont plus souvent le loisir d'assister à ce type de cours et qu'ils sont ensuite davantage susceptibles d'obtenir de meilleurs résultats que les élèves qui ne participent à aucun cours de soutien après la classe.

Écart de performance entre les élèves, selon les types de cours supplémentaires après la classe

Les élèves de milieux ou établissements défavorisés sur le plan socio-économique sont davantage susceptibles de suivre des cours supplémentaires en groupe.

$\square$ Les élèves de milieux ou établissements favorisés sur le plan socio-économique sont davantage susceptibles de suivre des cours supplémentaires en groupe.

$\square$ Pas de différence statistiquement significative entre les élèves de milieux et établissements favorisés ou défavorisés dans la participation à des cours supplémentaires en groupe.

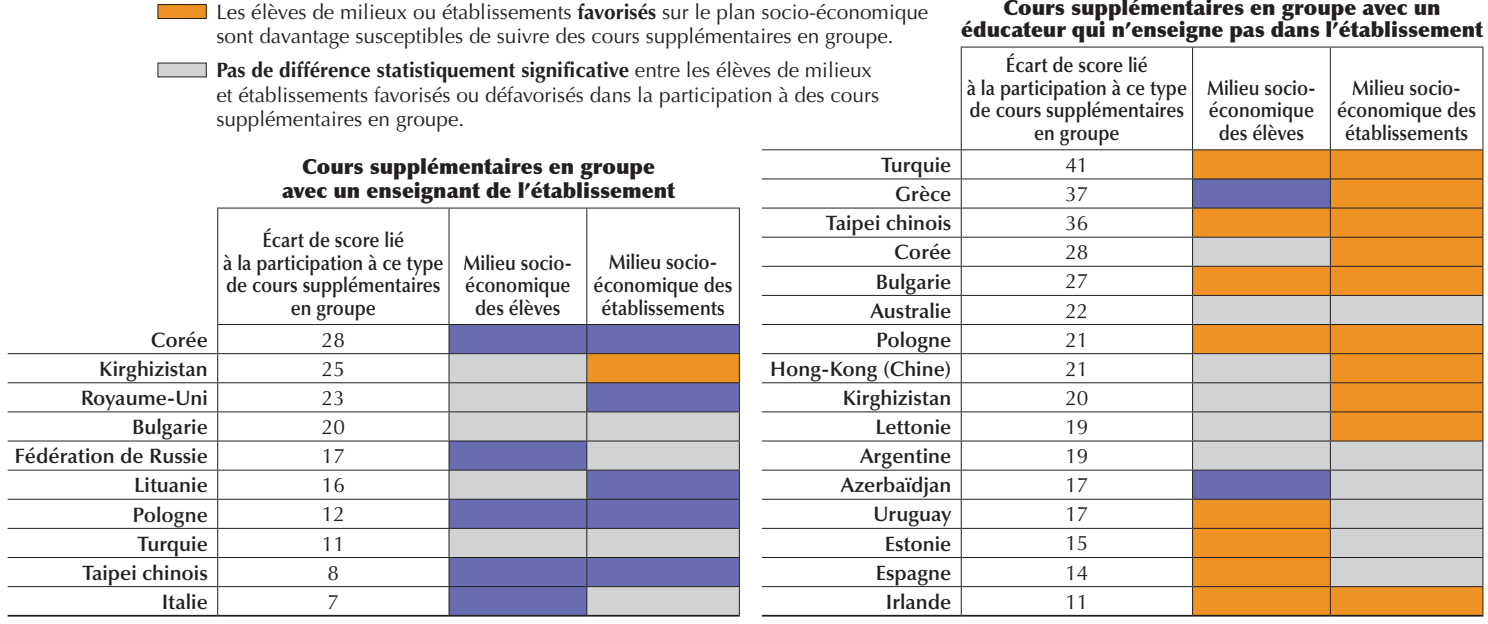

Cours supplémentaires en groupe avec un

Remarque : dans les tableaux, n’apparaissent que les pays où la participation à ce type de cours supplémentaires en groupe dispensés par des enseignants de l'établissement/d'autres éducateurs est positivement corrélée à la performance.

Source : Base de données PISA 2006 de l'OCDE.

Avant toute chose, les élèves doivent être convaincus de l'importance de ce qu'ils apprennent.
L'efficacité de l'apprentissage ne tient pas seulement aux ressources mises à la disposition des élèves; ceux-ci doivent eux-mêmes être convaincus de I'importance de bien réussir dans certaines matières. L'enquête PISA 2006 demandait aux élèves s'ils pensaient que bien réussir en sciences était important et ses résultats permettent de conclure que lorsqu'un élève pense de la sorte, l'avantage de performance consécutif à un nombre d'heures plus élevé en cours de sciences est supérieur à celui des élèves qui ne le pensent pas. 


\section{$P_{\text {ala Loupe }}$}

Pour les élèves qui déclarent qu'il n'est pas très important de bien réussir en sciences, l'ajout d'une heure de cours de sciences dans le cadre scolaire normal correspond à un avantage de score d'environ 22 points, après prise en compte du milieu socioéconomique de l'élève et de l'établissement. Il en découle une différence sensible de performance - s'élevant à 20 points de score au moins - entre ces élèves lorsqu'ils passent 5 heures ou plus par semaine dans les cours de sciences normaux. En Espagne, en France, en Grèce, en Islande, en Nouvelle-Zélande, au Portugal et en République tchèque, et dans les pays partenaires, en Roumanie, les attitudes envers I'apprentissage des sciences creusent un écart encore plus important. Les élèves de ces pays qui déclarent très

Relation entre la performance en sciences et le temps d'apprentissage passé dans les cours de sciences normaux dispensés dans le cadre scolaire, selon les attitudes des élèves vis-à-vis de l'importance d'avoir de bons résultats en sciences

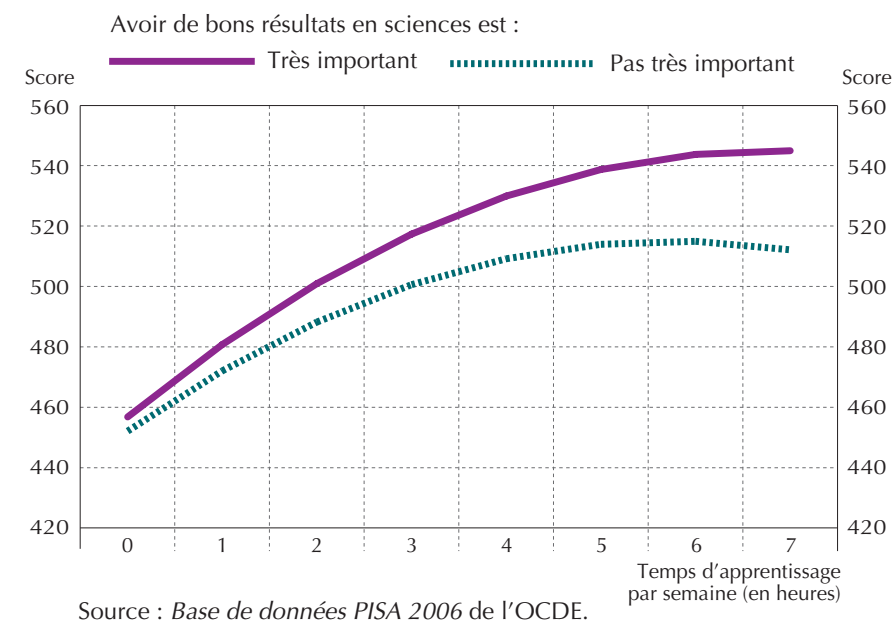

important d'avoir de bons résultats en sciences obtiennent, en moyenne, 6 points de plus pour chaque heure supplémentaire passée en cours de sciences que les élèves qui déclarent peu important d'avoir de bons résultats en sciences.

Il s'avère crucial d'optimiser le temps d'apprentissage, ainsi que la qualité de ce dernier, car il n'est souvent pas possible d'accroitre le nombre absolu d'heures d'apprentissage. Avant toute chose, il conviendrait de s'assurer que les élèves comprennent bien pourquoi il est important d'apprendre telle ou telle matière, ce qui, à terme, les aiderait à employer leur temps d'apprentissage plus efficacement.

Pour conclure : lorsqu'il s'agit d'apprentissage, c'est bien la qualité de l'apprentissage dans le cadre scolaire et les attitudes des élèves à l'égard de l'apprentissage qui comptent le plus, et non le nombre d'heures passées à étudier.

\section{Pour tout complément d'information}

Contacter Francesca Borgonovi (Francesa.Borgonovi@oecd.org), Miyako Ikeda (Miyako.Ikeda@oecd.org) et Soojin Park (Soojin.Park@oecd.org)

Consulter Quality Time for Students: Learning In and Out of School, disponible uniquement en anglais sur www.oecdbookshop.org

\section{Voir}

www.pisa.oecd.org 